\title{
Measurement of the inclusive charmless semileptonic branching fraction of beauty hadrons and a determination of $\left|V_{u b}\right|$ at LEP
}

\section{L3 Collaboration}

M. Acciarri aa, O. Adriani ${ }^{p}$, M. Aguilar-Benitez ${ }^{\text {z }}$, S. Ahlen ${ }^{\text {k }}$, J. Alcaraz ${ }^{\text {z }}$,

G. Alemanni ${ }^{\mathrm{v}}$, J. Allaby ${ }^{\mathrm{q}}$, A. Aloisio ${ }^{\mathrm{ac}}$, M.G. Alviggi ${ }^{\mathrm{ac}}$, G. Ambrosi ${ }^{\mathrm{s}}$, H. Anderhub av, V.P. Andreev ak, T. Angelescu ${ }^{\mathrm{m}}$, F. Anselmo ${ }^{\mathrm{i}}$, A. Arefiev ${ }^{\mathrm{ab}}$, T. Azemoon ${ }^{\text {c }}$, T. Aziz ${ }^{\mathrm{j}}$, P. Bagnaia ${ }^{\text {aj }}$, L. Baksay ${ }^{\text {aq }}$, R.C. Ball ${ }^{\mathrm{c}}$, S. Banerjee ${ }^{\mathrm{j}}$, Sw. Banerjee ${ }^{\mathrm{j}}, \mathrm{K}$. Banicz as, $\mathrm{A}$. Barczyk ${ }^{\text {av,at }}$, R. Barillère ${ }^{\mathrm{q}}, \mathrm{L}$. Barone ${ }^{\text {aj, }}$, P. Bartalini v , A. Baschirotto ${ }^{\text {aa }}$, M. Basile ${ }^{\text {i }, ~ R . ~ B a t t i s t o n ~}{ }^{\text {ag }}$, A. Bay ${ }^{\text {v }, ~ F . ~ B e c a t t i n i ~}{ }^{\text {p }}$, U. Becker ${ }^{\circ}$, F. Behner ${ }^{\text {av }}$, J. Berdugo ${ }^{\text {z }}$, P. Berges ${ }^{\circ}$, B. Bertucci ${ }^{\text {ag }}$, B.L. Betev ${ }^{\text {av }}$, S. Bhattacharya ${ }^{\mathrm{j}}$, M. Biasini ${ }^{\mathrm{ag}}$, A. Biland ${ }^{\text {av }}$, G.M. Bilei ${ }^{\mathrm{ag}}$, J.J. Blaising ${ }^{\mathrm{d}}$, S.C. Blyth ${ }^{\text {ah }}$, G.J. Bobbink ${ }^{\text {b }}$, R. Bock a , A. Böhm a , L. Boldizsar ${ }^{\text {n }}$, B. Borgia ${ }^{\text {q.aj }}$,

D. Bourilkov av, M. Bourquin ${ }^{\text {s }}$, D. Boutigny ${ }^{\mathrm{d}}$, S. Braccini ${ }^{\mathrm{s}}$, J.G. Branson ${ }^{\mathrm{am}}$, V. Brigljevic av , I.C. Brock ${ }^{\text {ah }}$, A. Buffini ${ }^{\text {p}}$, A. Buijs ${ }^{\text {ar }}$, J.D. Burger ${ }^{\circ}$, W.J. Burger ${ }^{\text {ag }}$, J. Busenitz ${ }^{\text {aq }}$, X.D. Cai ${ }^{\circ}$, M. Campanelli av ${ }^{\text {, }}$ M. Capell ${ }^{\circ}$, G. Cara Romeo ${ }^{\mathrm{i}}$, G. Carlino ${ }^{\text {ac }}$, A.M. Cartacci ${ }^{\text {p }}$ J. Casaus ${ }^{\text {z }}$, G. Castellini ${ }^{\text {p }}$, F. Cavallari ${ }^{\text {aj, }}$ N. Cavallo ${ }^{\text {ac }}$, C. Cecchi ${ }^{\mathrm{s}}$, M. Cerrada ${ }^{\mathrm{z}}$, F. Cesaroni ${ }^{\mathrm{w}}$, M. Chamizo ${ }^{\mathrm{z}}$, Y.H. Chang ax, U.K. Chaturvedi ${ }^{\mathrm{r}}$, M. Chemarin ${ }^{\mathrm{y}}$, A. Chen ${ }^{\text {ax }}$, G. Chen ${ }^{\mathrm{g}}$, G.M. Chen ${ }^{\mathrm{g}}$, H.F. Chen ${ }^{\mathrm{t}}$ H.S. Chen ${ }^{\mathrm{g}}$, M. Chen ${ }^{\circ}$, G. Chiefari ${ }^{\text {ac }}$, C.Y. Chien ${ }^{\mathrm{e}}$,

L. Cifarelli ${ }^{\text {al }}$, F. Cindolo ${ }^{\text {i }}$, C. Civinini ${ }^{\text {p }}$, I. Clare ${ }^{\circ}$, R. Clare ${ }^{\circ}$, G. Coignet ${ }^{d}$, A.P. Colijn ${ }^{\text {b }}$, N. Colino ${ }^{z}$, S. Costantini ${ }^{\text {h}}$, F. Cotorobai ${ }^{m}$, B. de la Cruz ${ }^{z}$, A. Csilling ${ }^{n}$, T.S. Dai ${ }^{\circ}$, R. D'Alessandro ${ }^{p}$, R. de Asmundis ${ }^{\text {ac }}$, A. Degré ${ }^{\mathrm{d}}$, K. Deiters ${ }^{\text {at }}$, P. Denes ai, F. DeNotaristefani aj, M. Diemoz aj, D. van Dierendonck ${ }^{\text {b }}$, F. Di Lodovico av, C. Dionisi ${ }^{\text {a,aj }}$, M. Dittmar ${ }^{\text {av }}$, A. Dominguez am, A. Doria ac, M.T. Dova ${ }^{\text {r, },}$, E. Drago ${ }^{\text {ac }}$, D. Duchesneau ${ }^{\text {d }}$, P. Duinker ${ }^{\text {b }}$, I. Duran ${ }^{\text {an }}$, S. Easo ag, H. El Mamouni ${ }^{y}$, A. Engler ah, F.J. Eppling o , F.C. Erné ${ }^{b}$, J.P. Ernenwein ${ }^{\mathrm{y}}$, P. Extermann ${ }^{\mathrm{s}}$, M. Fabre ${ }^{\text {at }}$, R. Faccini aj, M.A. Falagan ${ }^{\mathrm{z}}$, S. Falciano ${ }^{\text {aj }}$, A. Favara ${ }^{\mathrm{p}}$, J. Fay ${ }^{y}$, O. Fedin ak, M. Felcini av, T. Ferguson ${ }^{\text {ah }}$, F. Ferroni ${ }^{\text {aj, H. Fesefeldt a }}$, E. Fiandrini ${ }^{\text {ag }}$, J.H. Field ${ }^{\mathrm{s}}$, F. Filthaut ${ }^{\mathrm{q}}$, P.H. Fisher ${ }^{\circ}$, I. Fisk ${ }^{\text {am }}$, G. Forconi ${ }^{\circ}$, L. Fredj ${ }^{\text {s, K. Freudenreich }}{ }^{\text {av }}$, C. Furetta ${ }^{\text {aa }}$, Yu. Galaktionov ${ }^{\text {ab,o }}$, S.N. Ganguli ${ }^{j}$, 
P. Garcia-Abia ${ }^{\text {f }}$, M. Gataullin af ${ }^{\text {a }}$ S.S. Gau ${ }^{1}$, S. Gentile ${ }^{\text {aj }}$, J. Gerald ${ }^{\text {e, }}$ N. Gheordanescu ${ }^{\mathrm{m}}$, S. Giagu ${ }^{\text {aj }, ~ S . ~ G o l d f a r b ~}{ }^{\mathrm{v}}$, J. Goldstein ${ }^{\mathrm{k}}$, Z.F. Gong ${ }^{\mathrm{t}}$, A. Gougas ${ }^{\text {e }}$, G. Gratta ${ }^{\text {af }}$, M.W. Gruenewald ${ }^{\text {h }}$, R. van Gulik ${ }^{\text {b }, ~ V . K . ~ G u p t a ~}{ }^{\text {ai }}$, A. Gurtu ${ }^{\text {j }}$, L.J. Gutay ${ }^{\text {as }}$, D. Haas ${ }^{\text {f }}$, B. Hartmann ${ }^{\text {a }}$, A. Hasan ${ }^{\text {ad }}$, D. Hatzifotiadou ${ }^{\text {i }}$, T. Hebbeker ${ }^{\text {h }}$, A. Hervé ${ }^{\text {q }}$, P. Hidas ${ }^{\text {n }}$, J. Hirschfelder ${ }^{\text {ah }}$, W.C. van Hoek ${ }^{\text {ae }}$, H. Hofer ${ }^{\text {av }}$, H. Hoorani ah, S.R. Hou ${ }^{\text {ax }}$, G. Hu ${ }^{\text {e }}$, I. Iashvili au, B.N. Jin ${ }^{\text {g }}$, L.W. Jones ${ }^{\text {c }}$, P. de Jong ${ }^{\text {q }}$, I. Josa-Mutuberria ${ }^{\text {z }}$, A. Kasser ${ }^{\text {v }}$, R.A. Khan ${ }^{r}$, D. Kamrad au ${ }^{\text {a }}$ J.S. Kapustinsky ${ }^{x}$, Y. Karyotakis ${ }^{d}$, M. Kaur ${ }^{\text {r,2 }}{ }^{\text {, }}$ M.N. Kienzle-Focacci ${ }^{\text {s }}$, D. Kim ${ }^{\text {aj }}$, D.H. Kim ${ }^{\text {ap }}$, J.K. Kim ${ }^{\text {ap }}$, S.C. Kim ${ }^{\text {ap }}$, W.W. Kinnison ${ }^{\mathrm{x}}$, A. Kirkby ${ }^{\text {af }}$, D. Kirkby ${ }^{\text {af }}$, J. Kirkby ${ }^{\mathrm{q}}$, D. Kiss ${ }^{\mathrm{n}}$, W. Kittel ${ }^{\text {ae }}$, A. Klimentov ${ }^{\text {o,ab }}$, A.C. König ${ }^{\text {ae }}$, A. Kopp ${ }^{\text {au }}$, I. Korolko ${ }^{\text {ab }}$, V. Koutsenko ${ }^{\text {o,ab }}$, R.W. Kraemer ${ }^{\text {ah }}$, W. Krenz ${ }^{\text {a }}$, A. Kunin ${ }^{\text {o,ab }}$, P. Lacentre ${ }^{\text {au, }, ~}$, P. Ladron de Guevara ${ }^{\mathrm{z}}$, G. Landi ${ }^{\mathrm{p}}$, C. Lapoint ${ }^{\circ}$, K. Lassila-Perini ${ }^{\text {av }}$, P. Laurikainen ${ }^{\mathrm{u}}$, A. Lavorato ${ }^{\text {al }}$, M. Lebeau ${ }^{\mathrm{q}}$, A. Lebedev ${ }^{\circ}$, P. Lebrun ${ }^{\mathrm{y}}$, P. Lecomte ${ }^{\mathrm{av}}$, P. Lecoq ${ }^{\mathrm{q}}$, P. Le Coultre ${ }^{\text {av }}$, H.J. Lee ${ }^{\text {h }}$, C. Leggett ${ }^{\text {c }}$, J.M. Le Goff ${ }^{\mathrm{q}}$, R. Leiste ${ }^{\text {au }}$, E. Leonardi aj, P. Levtchenko ${ }^{\text {ak }}$, C. Li ${ }^{t}$, C.H. Lin ${ }^{\text {ax }}$, W.T. Lin ${ }^{a x}$, F.L. Linde ${ }^{\text {b, }}$, L. Lista ${ }^{\text {ac }}$, Z.A. Liu ${ }^{\mathrm{g}}$, W. Lohmann ${ }^{\text {au }}$, E. Longo ${ }^{\text {aj }}$, W. Lu ${ }^{\text {af }}$, Y.S. Lu ${ }^{\mathrm{g}}$, K. Lübelsmeyer ${ }^{\mathrm{a}}$, C. Luci ${ }^{\text {q,aj }}{ }^{\text {, D. Luckey }}{ }^{\circ}$, L. Luminari aj ${ }^{\text {aj }}$ W. Lustermann ${ }^{\text {av }}$, W.G. Ma ${ }^{t}$, M. Maity ${ }^{j}$, G. Majumder ${ }^{j}$, L. Malgeri ${ }^{\mathrm{q}}$, A. Malinin ${ }^{\mathrm{ab}}$, C. Maña ${ }^{\mathrm{z}}$, D. Mangeol ae,

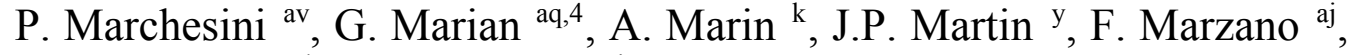
G.G.G. Massaro ${ }^{\text {b }}$, K. Mazumdar ${ }^{\text {j }}$, S. Mele ${ }^{\mathrm{q}}$, L. Merola ${ }^{\text {ac }}$, M. Meschini ${ }^{\mathrm{p}}$, W.J. Metzger ${ }^{\text {ae }}$, M. von der Mey ${ }^{\text {a }}$, Y. Mi ${ }^{\mathrm{v}}$, D. Migani ${ }^{\mathrm{i}}$, A. Mihul ${ }^{\mathrm{m}}$, A.J.W. van Mil ${ }^{\text {ae }}$, H. Milcent ${ }^{\mathrm{q}}$, G. Mirabelli aj, J. Mnich ${ }^{\mathrm{q}}$, P. Molnar ${ }^{\mathrm{h}}$, B. Monteleoni ${ }^{\mathrm{p}}$, R. Moore ${ }^{\mathrm{c}}$, T. Moulik ${ }^{\mathrm{j}}$, R. Mount ${ }^{\text {af }}$, F. Muheim ${ }^{\mathrm{s}}$, A.J.M. Muijs ${ }^{\mathrm{b}}$, S. Nahn ${ }^{\circ}$, M. Napolitano ${ }^{\text {ac }}$, F. Nessi-Tedaldi av, H. Newman ${ }^{\text {af }}$, T. Niessen ${ }^{\text {a }}$, A. Nippe ${ }^{\text {v }}$, A. Nisati ${ }^{\text {aj }}$, H. Nowak au, Y.D. Oh ${ }^{\text {ap }}$, G. Organtini aj, R. Ostonen ${ }^{\text {u }}$, S. Palit ${ }^{\text {l }}$, C. Palomares ${ }^{\text {z }}$, D. Pandoulas ${ }^{\text {a }}$, S. Paoletti aj,q, P. Paolucci ${ }^{\text {ac }}$, H.K. Park ${ }^{\text {ah }}$, I.H. Park ${ }^{\text {ap }}$, G. Pascale ${ }^{\text {aj }}$, G. Passaleva ${ }^{\text {q }}$, S. Patricelli ${ }^{\text {ac }}$, T. Paul ${ }^{1}$, M. Pauluzzi ${ }^{\text {ag }}$, C. Paus ${ }^{\text {q }}$, F. Pauss ${ }^{\text {av }}$, D. Peach ${ }^{\text {q }}$, Y.J. Pei ${ }^{\text {a }}$, S. Pensotti ${ }^{\text {aa }}$, D. Perret-Gallix ${ }^{\text {d, }}$ B. Petersen ${ }^{\text {ae }}$, S. Petrak ${ }^{\text {h }}$, A. Pevsner ${ }^{\mathrm{e}}$, D. Piccolo ${ }^{\mathrm{ac}}$, M. Pieri ${ }^{\mathrm{p}}$, P.A. Piroué ai, E. Pistolesi ${ }^{\text {aa }}$, V. Plyaskin ${ }^{\text {ab }}$, M. Pohl av, V. Pojidaev ${ }^{\text {ab,p }}$, H. Postema ${ }^{\circ}$, J. Pothier ${ }^{\mathrm{q}}$,

N. Produit ${ }^{\text {s }}$, D. Prokofiev ak ${ }^{\text {, }}$ J. Quartieri ${ }^{\text {al }}$, G. Rahal-Callot av, N. Raja ${ }^{\text {j, }}$, P.G. Rancoita ${ }^{\text {aa }}$, D. Rathje ${ }^{\mathrm{q}}$, M. Rattaggi ${ }^{\text {aa }}$, G. Raven ${ }^{\mathrm{am}}$, P. Razis ${ }^{\text {ad }}$, D. Ren ${ }^{\text {av }}$, M. Rescigno aj, S. Reucroft ${ }^{1}$, T. van Rhee ${ }^{\text {ar }}$, S. Riemann ${ }^{\text {au }}$, K. Riles ${ }^{\text {c }}$, O. Rind ${ }^{\text {c }}$, A. Robohm ${ }^{\text {av }}$, J. Rodin ${ }^{\text {aq }}$, B.P. Roe ${ }^{\text {c }}$, L. Romero ${ }^{\text {z }, ~ S . ~ R o s i e r-L e e s ~}{ }^{\text {d }}$, Ph. Rosselet ${ }^{\text {v }}$, S. Roth ${ }^{\text {a }}$, J.A. Rubio ${ }^{\text {q, D. Ruschmeier }}{ }^{\text {h }}$, H. Rykaczewski av, J. Salicio ${ }^{\text {q, }}$, E. Sanchez ${ }^{\text {z }}$, M.P. Sanders ${ }^{\text {ae }}$, M.E. Sarakinos ${ }^{\text {u }}$, G. Sauvage ${ }^{d}$, C. Schäfer ${ }^{\text {a }}$, V. Schegelsky ${ }^{\text {ak }}$, S. Schmidt-Kaerst a, D. Schmitz ${ }^{\text {a }}$, M. Schneegans ${ }^{\text {d}}$, N. Scholz av, 
H. Schopper ${ }^{\text {aw }}$, D.J. Schotanus ${ }^{\text {ae }}$, J. Schwenke ${ }^{\text {a }}$, G. Schwering a , C. Sciacca ${ }^{\text {ac }}$, D. Sciarrino ${ }^{\text {s, L. Servoli ag }}{ }^{\text {, S. Shevchenko }}{ }^{\text {af }}$, N. Shivarov ${ }^{\text {ao }}$, V. Shoutko ${ }^{\text {ab }}$, J. Shukla ${ }^{x}$, E. Shumilov ${ }^{\text {ab }}$, A. Shvorob ${ }^{\text {af }}$, T. Siedenburg ${ }^{\text {a }}$, D. Son ${ }^{\text {ap }}$, V. Soulimov ${ }^{\text {ac }}$, B. Smith ${ }^{\circ}$, P. Spillantini ${ }^{\mathrm{p}}$, M. Steuer ${ }^{\circ}$, D.P. Stickland ${ }^{\text {ai }}$, H. Stone ai , B. Stoyanov ${ }^{\text {ao }}$, A. Straessner a , K. Sudhakar ${ }^{\text {j, G. Sultanov }}{ }^{\text {r }}$, L.Z. Sun ${ }^{\text {t }}$, G.F. Susinno ${ }^{\text {s, H. Suter }}{ }^{\text {av }}$, J.D. Swain ${ }^{\text {r }}$, X.W. Tang ${ }^{\text {g }}$, L. Tauscher ${ }^{\text {f }}$, L. Taylor ${ }^{1}$, C. Timmermans ${ }^{\text {ae }}$, Samuel C.C. Ting ${ }^{\circ}$, S.M. Ting ${ }^{\circ}$, S.C. Tonwar ${ }^{j}$, J. Tóth ${ }^{n}$, C. Tully ${ }^{\text {ai }}$, K.L. Tung ${ }^{\mathrm{g}}$, Y. Uchida ${ }^{\circ}$, J. Ulbricht ${ }^{\text {av }}$, E. Valente ${ }^{\text {aj }}$, G. Vesztergombi ${ }^{\mathrm{n}}$, I. Vetlitsky ${ }^{\text {ab }}$, G. Viertel ${ }^{\text {av }}$, M. Vivargent ${ }^{d}$, S. Vlachos ${ }^{f}$, H. Vogel ${ }^{\text {ah }}$, H. Vogt ${ }^{\text {au }}$, I. Vorobiev ${ }^{\text {q,ab }}$, A.A. Vorobyov ${ }^{\text {ak }}$, A. Vorvolakos ${ }^{\text {ad }}$, M. Wadhwa ${ }^{\text {f }}$, W. Wallraff ${ }^{\text {a }}$, J.C. Wang ${ }^{\circ}$, X.L. Wang ${ }^{\mathrm{t}}$, Z.M. Wang ${ }^{\mathrm{t}}$, A. Weber ${ }^{\mathrm{a}}$, S.X. Wu ${ }^{\mathrm{o}}$, S. Wynhoff ${ }^{\mathrm{a}}$, J. Xu ${ }^{\mathrm{k}}$, Z.Z. Xu ${ }^{\mathrm{t}}$, B.Z. Yang ${ }^{\mathrm{t}}$, C.G. Yang ${ }^{\mathrm{g}}$, H.J. Yang ${ }^{\mathrm{g}}, \mathrm{M}$. Yang ${ }^{\mathrm{g}}$, J.B. Ye ${ }^{\mathrm{t}}$, S.C. Yeh ay , J.M. You ${ }^{\text {ah }}$, An. Zalite ${ }^{\text {ak }}$, Yu. Zalite ${ }^{\text {ak }}$, P. Zemp ${ }^{\text {av }}$, Y. Zeng ${ }^{\text {a }}$, Z.P. Zhang ${ }^{\text {t }}$, B. Zhou ${ }^{\text {k, }}$ Y. Zhou ${ }^{\text {c }}$, G.Y. Zhu ${ }^{\text {g }}$, R.Y. Zhu ${ }^{\text {af }}$, A. Zichichi ${ }^{\text {i,q,r, }}$, F. Ziegler ${ }^{\text {au }}$, G. Zilizi aq,4

${ }^{a}$ I. Physikalisches Institut, RWTH, D-52056 Aachen, FRG ${ }^{5}$

III. Physikalisches Institut, RWTH, D-52056 Aachen, FRG ${ }^{5}$

${ }^{\mathrm{b}}$ National Institute for High Energy Physics, NIKHEF, and University of Amsterdam, NL-1009 DB Amsterdam, The Netherlands c University of Michigan, Ann Arbor, MI 48109, USA

' Laboratoire d'Annecy-le-Vieux de Physique des Particules, LAPP, IN2P3-CNRS, BP 110, F-74941 Annecy-le-Vieux CEDEX, France

e Johns Hopkins University, Baltimore, MD 21218, USA

${ }^{\mathrm{f}}$ Institute of Physics, University of Basel, $\mathrm{CH}-4056$ Basel, Switzerland

${ }^{\mathrm{g}}$ Institute of High Energy Physics, IHEP, 100039 Beijing, China ${ }^{6}$

h Humboldt University, D-10099 Berlin, FRG ${ }^{5}$

${ }^{\mathrm{i}}$ University of Bologna and INFN-Sezione di Bologna, I-40126 Bologna, Italy

${ }^{\mathrm{j}}$ Tata Institute of Fundamental Research, Bombay 400 005, India

${ }^{\mathrm{k}}$ Boston University, Boston, MA 02215, USA

${ }^{1}$ Northeastern University, Boston, MA 02115, USA

${ }^{\mathrm{m}}$ Institute of Atomic Physics and University of Bucharest, R-76900 Bucharest, Romania

${ }^{\mathrm{n}}$ Central Research Institute for Physics of the Hungarian Academy of Sciences, H-1525 Budapest 114, Hungary ${ }^{7}$

${ }^{\circ}$ Massachusetts Institute of Technology, Cambridge, MA 02139, USA

${ }^{\mathrm{p}}$ INFN Sezione di Firenze and University of Florence, I-50125 Florence, Italy

${ }^{\mathrm{q}}$ European Laboratory for Particle Physics, CERN, CH-1211 Geneva 23, Switzerland

${ }^{\mathrm{r}}$ World Laboratory, FBLJA Project, CH-1211 Geneva 23, Switzerland

${ }^{\mathrm{s}}$ University of Geneva, CH-1211 Geneva 4, Switzerland

${ }^{\mathrm{t}}$ Chinese University of Science and Technology, USTC, Hefei, Anhui 230 029, China ${ }^{6}$

" SEFT, Research Institute for High Energy Physics, P.O. Box 9, SF-00014 Helsinki, Finland

${ }^{v}$ University of Lausanne, CH-1015 Lausanne, Switzerland

${ }^{\mathrm{w}}$ INFN-Sezione di Lecce and Universitá Degli Studi di Lecce, I-73100 Lecce, Italy

x Los Alamos National Laboratory, Los Alamos, NM 87544, USA

${ }^{\mathrm{y}}$ Institut de Physique Nucléaire de Lyon, IN2P3-CNRS, Université Claude Bernard, F-69622 Villeurbanne, France

${ }^{\mathrm{z}}$ Centro de Investigaciones Energeticas, Medioambientales y Tecnologicas, CIEMAT, E-28040 Madrid, Spain ${ }^{8}$

${ }^{\text {aa } I N F N-S e z i o n e ~ d i ~ M i l a n o, ~ I-20133 ~ M i l a n, ~ I t a l y ~}$

ab Institute of Theoretical and Experimental Physics, ITEP, Moscow, Russia

${ }^{\text {ac } I N F N-S e z i o n e ~ d i ~ N a p o l i ~ a n d ~ U n i v e r s i t y ~ o f ~ N a p l e s, ~ I-80125 ~ N a p l e s, ~ I t a l y ~}$

ad Department of Natural Sciences, University of Cyprus, Nicosia, Cyprus

${ }^{\text {ae }}$ University of Nijmegen and NIKHEF, NL-6525 ED Nijmegen, The Netherlands

${ }^{\text {af }}$ California Institute of Technology, Pasadena, CA 91125, USA

ag INFN-Sezione di Perugia and Universitá Degli Studi di Perugia, I-06100 Perugia, Italy 
${ }^{\text {ah }}$ Carnegie Mellon University, Pittsburgh, PA 15213, USA

ai Princeton University, Princeton, NJ 08544, USA

aj INFN-Sezione di Roma and University of Rome, "La Sapienza", I-00185 Rome, Italy

${ }^{\mathrm{ak}}$ Nuclear Physics Institute, St. Petersburg, Russia

${ }^{\text {al }}$ University and INFN, Salerno, I-84100 Salerno, Italy

am University of California, San Diego, CA 92093, USA

${ }^{a n}$ Dept. de Fisica de Particulas Elementales, Univ. de Santiago, E-15706 Santiago de Compostela, Spain

ao Bulgarian Academy of Sciences, Central Lab. of Mechatronics and Instrumentation, BU-1113 Sofia, Bulgaria

${ }^{\text {ap }}$ Center for High Energy Physics, Adv. Inst. of Sciences and Technology, 305-701 Taejon, South Korea

${ }^{\text {aq }}$ University of Alabama, Tuscaloosa, AL 35486, USA

${ }^{\text {ar }}$ Utrecht University and NIKHEF, NL-3584 CB Utrecht, The Netherlands

${ }^{\text {as }}$ Purdue University, West Lafayette, IN 47907, USA

at Paul Scherrer Institut, PSI, CH-5232 Villigen, Switzerland

${ }^{\text {au }}$ DESY-Institut für Hochenergiephysik, D-15738 Zeuthen, FRG

av Eidgenössische Technische Hochschule, ETH Zürich, CH-8093 Zürich, Switzerland

${ }^{\text {aw }}$ University of Hamburg, D-22761 Hamburg, FRG

${ }^{a x}$ National Central University, Chung-Li, Taiwan, China

ay Department of Physics, National Tsing Hua University, Taiwan, China

Received 15 June 1998

Editor: K. Winter

\section{Abstract}

A measurement of the inclusive charmless semileptonic branching fraction of beauty hadrons, $\mathrm{b} \rightarrow \mathrm{X}_{\mathrm{u}} \ell \nu$, has been performed using almost two million hadronic $\mathrm{Z}$ decays collected by the L3 experiment at LEP, yielding the result: $\mathrm{Br}\left(\mathrm{b} \rightarrow \mathrm{X}_{\mathrm{u}} \ell \nu\right)=(3.3 \pm 1.0 \pm 1.7) \times 10^{-3}$. The first uncertainty is statistical and the second is systematic. The modulus of the Cabibbo-Kobayashi-Maskawa matrix element $\mathrm{V}_{\mathrm{ub}}$ extracted from this measurement is: $\left|\mathrm{V}_{\mathrm{ub}}\right|=\left(6.0_{-1.0-1.9}^{+0.8} \pm 0.2\right) \times$ $10^{-3}$, where the uncertainties are statistical, systematic and theoretical, respectively. (C) 1998 Elsevier Science B.V. All rights reserved.

\section{Introduction}

The Cabibbo-Kobayashi-Maskawa [1,2] (CKM) matrix describes the mixing of the quark mass eigenstates with the weak interaction ones. The measure-

\footnotetext{
${ }^{1}$ Also supported by CONICET and Universidad Nacional de La Plata, CC 67, 1900 La Plata, Argentina.

${ }^{2}$ Also supported by Panjab University, Chandigarh-160014, India.

${ }^{3}$ Supported by Deutscher Akademischer Austauschdienst.

${ }^{4}$ Also supported by the Hungarian OTKA fund under contract numbers T22238 and T026178.

${ }^{5}$ Supported by the German Bundesministerium für Bildung, Wissenschaft, Forschung und Technologie

${ }^{6}$ Supported by the National Natural Science Foundation of China.

${ }^{7}$ Supported by the Hungarian OTKA fund under contract numbers T019181, F023259 and T024011.

${ }^{8}$ Supported also by the Comisión Interministerial de Ciencia y Technología.
}

ment of its elements is of fundamental interest for the description of the charged current part of the Standard Model Lagrangian [3-5]. This $3 \times 3$ unitary matrix can be written [6] in terms of only four real parameters, two of which, $\rho$ and $\eta$, are poorly known:

$$
\begin{aligned}
& \mathrm{V}_{\mathrm{CKM}} \\
& =\left(\begin{array}{ccc}
\mathrm{V}_{\mathrm{ud}} & \mathrm{V}_{\mathrm{us}} & \mathrm{V}_{\mathrm{ub}} \\
\mathrm{V}_{\mathrm{cd}} & \mathrm{V}_{\mathrm{cs}} & \mathrm{V}_{\mathrm{cb}} \\
\mathrm{V}_{\mathrm{td}} & \mathrm{V}_{\mathrm{ts}} & \mathrm{V}_{\mathrm{tb}}
\end{array}\right) \\
& \simeq\left(\begin{array}{ccc}
1-\frac{\lambda^{2}}{2} & \lambda & A \lambda^{3}(\rho-i \eta) \\
-\lambda & 1-\frac{\lambda^{2}}{2} & A \lambda^{2} \\
A \lambda^{3}(1-\rho-i \eta) & -A \lambda^{2} & 1
\end{array}\right) .
\end{aligned}
$$

Here, powers higher than three in the parameter $\lambda$ are neglected. A determination of $\left|\mathrm{V}_{\mathrm{ub}}\right|$, combined with the knowledge of the CKM matrix elements 
related to the mixing in the neutral beauty and kaon systems, provides stringent limits on the possible values of $\rho$ and $\eta$ and helps to address an important open question of the Standard Model: the mechanism of the violation of CP symmetry.

Several measurements of $\left|\mathrm{V}_{\mathrm{ub}}\right|$ performed at the $\Upsilon(4 \mathrm{~S})$ exist to date. The CLEO [7,8] and ARGUS $[9,10]$ collaborations reported excesses in the lepton endpoint spectra in $\mathrm{B}^{9}$ decays, constituting the first evidence for $\mathrm{B} \rightarrow \mathrm{X}_{\mathrm{u}} \ell \nu{ }^{10}$ transitions. The CLEO collaboration $[11,12]$ has also reported the measurement of the exclusive $\mathrm{B} \rightarrow(\pi, \rho, \omega) \ell \nu$ transitions. Both these experimental approaches have a strong dependence on the models used to extract the value of the branching fractions involved and $\left|\mathrm{V}_{\mathrm{ub}}\right|$ itself.

At LEP, the boost of the $b$ hadron system and the good separation of the two initial state $b$ quarks make it feasible to study the inclusive $\mathrm{b} \rightarrow \mathrm{X}_{\mathrm{u}} \ell \nu$ transitions in a momentum range not restricted to the endpoint region, like as the $\Upsilon(4 \mathrm{~S})$, and measure the corresponding branching fraction. This measurement allows the determination of $\left|\mathrm{V}_{\mathrm{ub}}\right|$ with a theoretical uncertainty of approximately $4 \%[13,14]$. This approach has been recently exploited by the ALEPH collaboration [15].

This letter describes a study of $\mathrm{b} \rightarrow \mathrm{X}_{\mathrm{u}} \ell \nu$ transitions at LEP, the measurement of the branching fraction and the extraction of $\left|\mathrm{V}_{\mathrm{ub}}\right| .1 .8$ million hadronic $\mathrm{Z}$ decays collected at LEP in 1994 and 1995 by the L3 detector have been analysed. The detector, its subsystems and their performance are described in detail in Ref. [16-19].

\section{Event simulation}

Crucial to this analysis is the Monte Carlo simulation of the signal and background processes. A modified version of the JETSET 7.4 Monte Carlo [20,21], based on the Lund parton shower model, was used to

\footnotetext{
${ }^{9}$ The symbol $\mathrm{B}$ in this paper denotes the ensemble of $\mathrm{B}^{+}, \mathrm{B}^{-}$, $\mathrm{B}_{\mathrm{d}}^{0}$ and $\overline{\mathrm{B}}_{\mathrm{d}}^{0}$ mesons at $\sqrt{s}=\Upsilon(4 \mathrm{~S})$, with the addition of the two states $\mathrm{B}_{\mathrm{s}}^{0}$ and $\overline{\mathrm{B}}_{\mathrm{s}}^{0}$ at $\sqrt{s}=m_{\mathrm{Z}}$. The symbol b comprises all the hadrons containing a $\mathrm{b}$ quark.

${ }^{10}$ The symbols $\mathrm{X}_{\mathrm{c}}$ and $\mathrm{X}_{\mathrm{u}}$ are used to denote charmed and charmless hadronic systems, respectively, the latter containing a $\mathrm{u}$ quark; the symbol $\ell$ indicates either an electron or a muon.
}

generate a total of $200000 \mathrm{Z} \rightarrow \mathrm{b} \bar{b}$ events in the central region of the L3 detector. One $b$ quark was forced to hadronise and decay as either $\mathrm{B} \rightarrow \mathrm{X}_{\mathrm{u}} \mathrm{e} \nu_{e}$ or $\mathrm{B} \rightarrow \mathrm{X}_{\mathrm{u}} \mu \nu_{\mu}$, with equal probabilities, and the other one into any of the allowed final states. The lepton momentum spectrum for these $\mathrm{B} \rightarrow \mathrm{X}_{\mathrm{u}} \ell \nu$ transitions was generated according to the ACCMM [22] model with the parameters $p_{f}=298 \mathrm{MeV}$ and $m_{u}=150 \mathrm{MeV}$. The branching fraction of the exclusive transition to pions, $\operatorname{Br}(\mathrm{B} \rightarrow \pi \ell \nu) / \operatorname{Br}(\mathrm{B} \rightarrow$ $\left.\mathrm{X}_{\mathrm{u}} \ell \nu\right)$, was changed from the original value of 0.32 to the more realistic figure of $0.15[7,8,11,12]$. The pion momentum spectrum in the $\mathrm{B} \rightarrow \pi \ell \nu$ transitions was simulated according to Ref. [23].

The events were then passed through the full L3 simulation program which takes into account the effects of energy loss, multiple scattering, interactions and decays in the detector materials. This simulation is based on the GEANT package [24] with the GHEISHA [25] program for the simulation of hadronic interactions. Inefficiencies of the various sub-detectors, as obtained from the data, were also simulated. The simulated events, after reconstruction by the same program used for the data, were used to tune the analysis procedure and calculate the efficiency of the event selection criteria.

Background processes were studied using seven million Monte Carlo hadronic $\mathrm{Z}$ decays generated with the JETSET 7.4 code and passed through the same detector simulation and reconstruction chain described above. The hadronisation of the light quarks was described by the Lund symmetric fragmentation function [20,21], while the Peterson fragmentation function [26] was used to model the fragmentation of the $c$ and $b$ quarks. The ACCMM [22] model was used to describe the lepton momentum spectrum in the $\mathrm{b} \rightarrow \mathrm{X}_{\mathrm{c}} \ell \nu$ transitions using the parameters $p_{f}=298 \mathrm{MeV}$ and $m_{c}=1673 \mathrm{MeV}$ as suggested in Ref. [27]. The branching fraction of this process was fixed to $10.30 \%$; the transitions $\mathrm{b} \rightarrow \mathrm{c}$ $\rightarrow \ell$ and $\mathrm{b} \rightarrow \overline{\mathrm{c}} \rightarrow \ell$ were simulated with branching fractions of $8.0 \%$ and $1.3 \%$, respectively [27,28]. $\mathrm{B} \rightarrow \mathrm{X}_{\mathrm{u}} \ell \nu$ transitions in this sample comprised $0.15 \%$ of the B decays. Charmless semileptonic transitions of $b$ baryons were simulated neither in the signal nor in the background sample.

The mean value of the ratio of the energy of the weakly decaying $b$ hadron to the beam energy used 
in the generation of both the signal and background Monte Carlo samples was $\left\langle x_{\mathrm{E}}^{\mathrm{b}}\right\rangle=0.709$ [29]. The analogous parameter for the charmed hadrons was chosen to be $\left\langle x_{\mathrm{E}}^{\mathrm{c}}\right\rangle=0.480$.

\section{Analysis procedure}

The main background for the identification of $\mathrm{b} \rightarrow \mathrm{X}_{\mathrm{u}} \ell \nu$ transitions are the CKM favoured $\mathrm{b} \rightarrow$ $\mathrm{X}_{\mathrm{c}} \ell \nu$ decays, whose rate is larger by about two orders of magnitude. Other background sources are: hadronic $b$ decays to charmed hadrons which then undergo a semileptonic decay, hadronic $\mathrm{Z}$ decays to $\mathrm{c}$ or light quarks, and possible lepton misidentification in the full sample of hadronic $\mathrm{Z}$ decays. All these classes of background events are largely eliminated by the selection criteria devised to enhance the ratio between $\mathrm{b} \rightarrow \mathrm{X}_{\mathrm{u}} \ell \nu$ and $\mathrm{b} \rightarrow \mathrm{X}_{\mathrm{c}} \ell \nu$ events.

The main difference between $\mathrm{b} \rightarrow \mathrm{X}_{\mathrm{c}} \ell \nu$ and $\mathrm{b} \rightarrow$ $\mathrm{X}_{\mathrm{u}} \ell \nu$ decays is the large mass of the charmed system as compared to that of the charmless one. The lepton momentum, $p_{\ell}$, and its transverse momentum with respect to the jet axis, $p_{t}$, will thus be larger for leptons emitted together with a lighter charmless meson than in the case of a CKM favoured decay. Owing to the higher energy available for the hadronic system, the momentum, $p_{1}$, of the most energetic detected object, i.e., a charged track or an isolated electromagnetic cluster, will be on average larger for $\mathrm{b} \rightarrow \mathrm{X}_{\mathrm{u}} \ell \nu$ than for $\mathrm{b} \rightarrow \mathrm{X}_{\mathrm{c}} \ell \nu$. The opposite relation holds for the momentum of the second most energetic object, $p_{2}$. The combined system of this most energetic object and the lepton will be a better approximation to the $\mathrm{b}$ hadron in $\mathrm{b} \rightarrow \mathrm{X}_{\mathrm{u}} \ell \nu$ than in $\mathrm{b} \rightarrow \mathrm{X}_{\mathrm{c}} \ell \nu$ transitions, since less particles are missing in this approximation. As a consequence, the invariant mass, $m_{1 \ell}$, and total momentum, $p_{1 \ell}$, of this system will be on average smaller for $\mathrm{b} \rightarrow \mathrm{X}_{\mathrm{c}} \ell \nu$ than for $\mathrm{b} \rightarrow \mathrm{X}_{\mathrm{u}} \ell \nu$ decays. The different multiplicity of the final states, together with the multiplicity of the other fragmentation particles, will also reflect the differences described above. Thus, more objects will populate a cone of $30^{\circ}$ half-opening angle around the lepton, $N_{30^{\circ}}$, for $\mathrm{b} \rightarrow \mathrm{X}_{\mathrm{c}} \ell \nu$ than for $\mathrm{b} \rightarrow \mathrm{X}_{\mathrm{u}} \ell \nu$ transitions. The pseudo-rapidity, $\eta_{1}$, and the transverse momentum, $p_{t 1}^{\ell}$, of the most energetic object, both calculated with respect to the lepton direction, also help to discriminate the $\mathrm{b} \rightarrow \mathrm{X}_{\mathrm{u}} \ell \nu$ decays from the $\mathrm{b} \rightarrow \mathrm{X}_{\mathrm{c}} \ell \nu$ background.

The analysis procedure is the following: first, selection criteria have been devised to identify the electrons, muons, charged tracks and neutral clusters needed to form the above kinematic variables. A preselection intended to enhance $\mathrm{b} \rightarrow \mathrm{X}_{\mathrm{u}} \ell \nu$ type events has then been performed and, from the study of the signal and background Monte Carlo samples selected at this stage, final values of the cuts on the kinematic variables have been set. Two different and overlapping selections have been devised in order to explore the different phase space regions of the decay products of the $\mathrm{b} \rightarrow \mathrm{X}_{\mathrm{u}} \ell \nu$ transitions. The first made use of a criterion on $p_{1}$ to select a high momentum hadronic system. The second exploits the opposite situation of a soft hadronic system and a high energy lepton by means of selection criteria based on $p_{\ell}, p_{t 1}^{\ell}$ and $\eta_{1}$. In addition, a third selection based on the common features of the $\mathrm{b} \rightarrow \mathrm{X}_{\mathrm{u}} \ell \nu$ transitions as described by the variables $p_{t}, p_{2}, p_{1 \ell}$ and $m_{1 \ell}$ has been applied.

\section{Event selection}

Hadronic $\mathrm{Z}$ decays were first selected by requiring a high multiplicity and a high and well balanced visible energy, both in the longitudinal and transverse plane [30]. The selection requirements for the identification of tracks, clusters and leptons are summarised below.

- Tracks were reconstructed in the central tracking chamber requiring at least 30 hits with the a minimum distance between the first and the last of 40 wires. Two or more hits should be in the inner part of the tracker. Only tracks with a transverse momentum above $500 \mathrm{MeV}$, a total momentum below $30 \mathrm{GeV}$ and a distance of closest approach in the plane perpendicular to the beam smaller than $3 \mathrm{~mm}$ have been accepted ${ }^{11}$.

- Electromagnetic clusters were chosen from showers in the full angular coverage of the electromagnetic calorimeter by requiring an energy deposi-

\footnotetext{
${ }^{11}$ These additional criteria are not required to be fulfilled by the tracks used in the lepton identification described below.
} 
tion in excess of $100 \mathrm{MeV}$ in three or more neighbouring crystals, with less than $3 \mathrm{GeV}$, in a cone of $7^{\circ}$ half-opening angle in the hadron calorimeter. These showers had to be consistent with the expected behaviour of electromagnetic energy depositions. The isolation of these clusters was established by requiring the ratio of the energy depositions in a $3 \times 3$ to a $5 \times 5$ crystal matrix centred on the crystal of the cluster with the largest energy to be larger than 0.9 . No track was allowed to point to the cluster within an angle of $5 \mathrm{mrad}$ in the transverse plane.

- Muons were identified in the barrel region of the muon spectrometer which covers a polar angle range of $36^{\circ}$ to $144^{\circ}$. The reconstructed muon tracks had to point toward the event vertex both in the transverse and longitudinal planes. A track in the central tracker was required to be associated with the muon.

- Electrons were selected starting from the identification of the electromagnetic clusters described above, rejecting those in the end-caps or those with less than six crystals. A track was required to point to the cluster within an angle of $5 \mathrm{mrad}$ in the transverse plane. The transverse momentum of this track had to be compatible with the transverse energy of the cluster within four times the resolution on their difference.

Only events containing at least one lepton with a momentum above $3 \mathrm{GeV}$ and with a thrust axis

Table 1

The number of data and Monte Carlo selected hemispheres at different stages of the selection. The relative contributions to the background Monte Carlo and the signal efficiencies are also reported

\begin{tabular}{llll}
\hline & Stage 1 & Stage 2 & $\begin{array}{l}\text { Final } \\
\text { selection }\end{array}$ \\
\hline Data & 96568 & 11935 & 576 \\
Background Monte Carlo & 96122 & 11566 & 495 \\
$\mathrm{~b} \rightarrow \mathrm{X}_{\mathrm{c}} \ell \nu$ & $39.7 \%$ & $78.2 \%$ & $82.5 \%$ \\
$\mathrm{~b} \rightarrow \mathrm{c} \rightarrow \ell$ & $12.8 \%$ & $4.7 \%$ & $3.1 \%$ \\
$\mathrm{~b} \rightarrow \mathrm{c} \rightarrow \ell$ & $4.3 \%$ & $1.2 \%$ & $0.7 \%$ \\
Other b decays & $7.0 \%$ & $4.4 \%$ & $6.4 \%$ \\
$\mathrm{c} \rightarrow \ell$ & $18.1 \%$ & $4.6 \%$ & $2.6 \%$ \\
Others & $18.1 \%$ & $6.9 \%$ & $4.7 \%$ \\
$\varepsilon\left(\mathrm{B} \rightarrow \mathrm{X}_{\mathrm{u}} \mu \nu_{\mu}\right)$ & $32.6 \%$ & $12.1 \%$ & $1.3 \%$ \\
$\varepsilon\left(\mathrm{B} \rightarrow \mathrm{X}_{\mathrm{u}} \mathrm{e} \nu_{e}\right)$ & $23.3 \%$ & $11.5 \%$ & $1.7 \%$ \\
\hline
\end{tabular}

Table 2

The requirements for the different stages of the analysis and their variation interval for the systematic uncertainty studies. Definitions of the variables are given in the text

\begin{tabular}{llll}
\hline & Stage 2 & Final selection & Variation \\
\hline$p_{t}>1.5 \mathrm{GeV}$ & $2.8 \mathrm{GeV}$ & $2.4-3.1 \mathrm{GeV}$ \\
$p_{1}>-$ & $7.0 \mathrm{GeV}$ & $6.1-7.8 \mathrm{GeV}$ \\
$p_{2}<-$ & $4.3 \mathrm{GeV}$ & $2.8-5.9 \mathrm{GeV}$ \\
$p_{1 \ell}>13.5 \mathrm{GeV}$ & $17.3 \mathrm{GeV}$ & $16.5-18.0 \mathrm{GeV}$ \\
$m_{1 \ell} \in(1.6,7.5) \mathrm{GeV}$ & $(2.3,5.7) \mathrm{GeV}$ & $(2.1,5.9)-(2.5,5.6) \mathrm{GeV}$ \\
$p_{\ell}>-$ & $6.1 \mathrm{GeV}$ & $4.5-7.5 \mathrm{GeV}$ \\
$p_{t 1}^{\ell}>-$ & $2.8 \mathrm{GeV}$ & $2.5-3.1 \mathrm{GeV}$ \\
$\eta_{1}<-$ & 2.55 & $2.6-3.5$ \\
$N_{30^{\circ}}<9$ & - & - \\
\hline
\end{tabular}

pointing in the central region of the detector were selected. Each event was then divided into two hemispheres by the plane perpendicular to the thrust axis and the kinematic quantities described in the previous section were calculated for the hemispheres containing a lepton. Almost 100000 hemispheres in the data satisfy these requirements, as reported in the first column of Table 1 (Stage 1).

Only the most energetic lepton in the hemisphere has been taken into account and the clusters and the track associated with it were not included in the calculation of the kinematic variables used throughout this analysis.

The Monte Carlo simulation of hadronic $\mathrm{Z}$ decays was normalised to this number of lepton hemispheres and the $\mathrm{B} \rightarrow \mathrm{X}_{\mathrm{u}} \ell \nu$ transitions were then removed from it, giving a background Monte Carlo sample whose number of events is also shown in the first column of Table 1, together with its relative composition.

The stage 2 requirements on $p_{t}, p_{1 \ell}, m_{1 \ell}$ and $N_{30^{\circ}}$ listed in the first column of Table 2 have been applied to this sample in order to enhance its $b \rightarrow$ $\mathrm{X}_{\mathrm{u}} \ell \nu$ content. Fig. 1 shows the distributions of these four variables before the application of these cuts, which are also indicated. This selection reduces the data and background samples by a factor of nine, and the signal sample by only a factor of between two and three, as summarised in the second column of Table 1.

The distributions of the kinematic variables described in the previous section were studied and a set of final selection requirements based on them was 

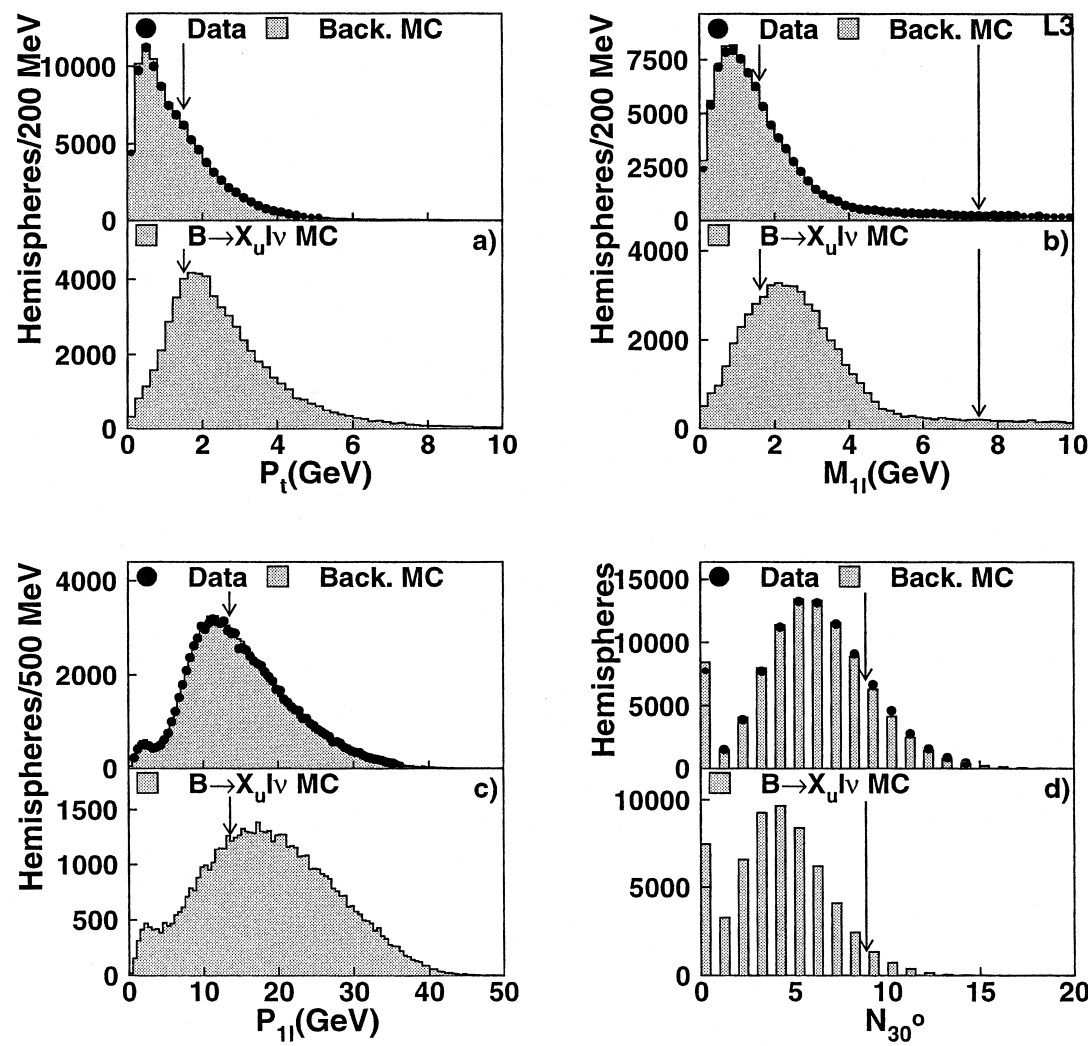

Fig. 1. Distributions at stage 1 of variables for data and Monte Carlo simulations of the background (top part of each figure) and for the expected signal (bottom part of each figure with arbitrary normalisation). a) Transverse momentum of the lepton, b) invariant mass of the lepton and the most energetic object, c) momentum sum of the lepton and the most energetic object and d) multiplicity of objects (see text) in a $30^{\circ}$ half-opening cone around the lepton. The arrows indicate the positions of the cuts used for stage 2 selection.

devised, as presented in the second column of Table 2. Fig. 2 displays some of these variables before the application of these final cuts, together with their value.

\section{Determination of the branching fraction}

After the application of the final selection criteria described in the previous section, 576 hemispheres are retained in data, while 495 are expected from the background Monte Carlo, normalised as described above. A total efficiency of $3.0 \%$ for the electron and muon modes was measured, as reported in the last column of Table 1, which also shows the relative background composition. These numbers, combined in a Poissonian likelihood, lead to a determination of the $\mathrm{b} \rightarrow \mathrm{X}_{\mathrm{u}} \ell \nu$ branching fraction as:

$\operatorname{Br}\left(\mathrm{b} \rightarrow \mathrm{X}_{\mathrm{u}} \ell \nu\right)=(3.3 \pm 1.0) \times 10^{-3}$,

where the uncertainty is due to data statistics only. In this calculation, the initial number of hadronic $Z$ decays is 1855152 , and the ratio of $Z$ boson decays to $b$ quarks relative to the hadronic $Z$ decays, $R_{b}$, is $0.2174 \pm 0.0009$ [28]. The separate results for electrons and muons with their statistical uncertainties are:

$\operatorname{Br}\left(\mathrm{b} \rightarrow \mathrm{X}_{\mathrm{u}} \mathrm{e} \nu_{\mathrm{e}}\right)=(3.6 \pm 1.3) \times 10^{-3}$ and $\operatorname{Br}\left(\mathrm{b} \rightarrow \mathrm{X}_{\mathrm{u}} \mu \nu_{\mu}\right)=(3.0 \pm 1.5) \times 10^{-3}$.

All the results above have been obtained with the assumption that the efficiency for semileptonic 

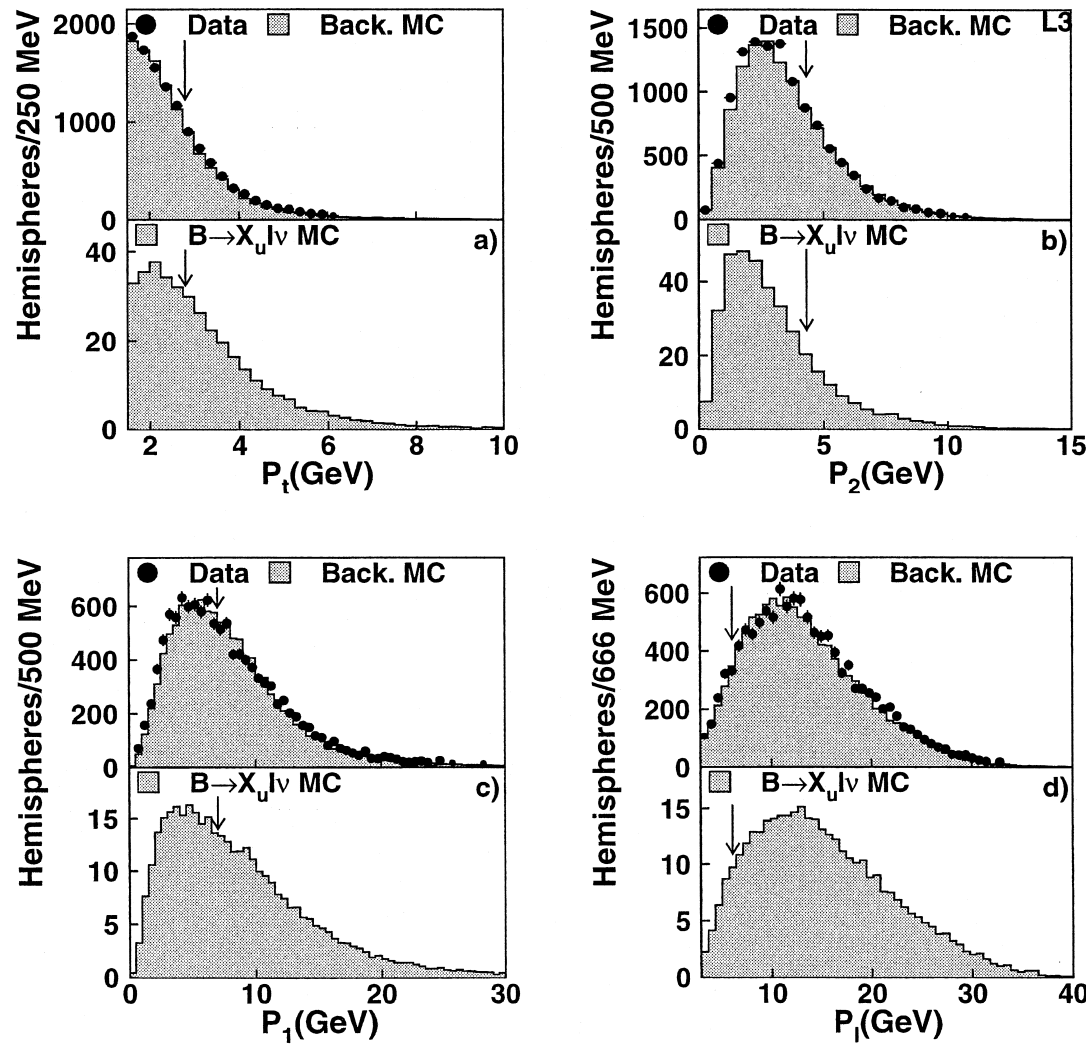

Fig. 2. Distributions of some selection variables for data and Monte Carlo simulations of the background (top part of each figure) and for the expected signal (bottom part of each figure, normalised to its measured branching fraction). Stage 2 criteria have been applied. a) Transverse momentum of the lepton, b) momentum of the second most energetic object, c) momentum of the most energetic object and d) lepton momentum. The variables shown in a) and b) are an example of the global kinematic selection, while the ones in c) and d) belong to the selection meant to enhance the two different phase space contributions described in the text. The arrows indicate the positions of the cuts used for the final selection.

charmless b baryon decays is equal to that for mesons, as calculated from the described signal Monte Carlo sample.

Fig. 3 shows the lepton momentum spectrum in the $\mathrm{B}$ rest frame for events passing the final selection in the $\mathrm{B} \rightarrow \mathrm{X}_{\mathrm{u}} \ell \nu$ Monte Carlo sample, which proves that this analysis is sensitive to a large fraction of the spectrum, in contrast to only the endpoint region for the experiments at the $\Upsilon(4 S)$.

\section{Study of systematic uncertainties}

The sources of systematic uncertainties can be classified into four categories: 1) uncertainties in the determination of the expected number of background events coming from both Monte Carlo statistics and modelling; 2) uncertainties in the calculation of the signal efficiency due to these sources; 3) uncertainties due to the background normalisation; 4) uncertainties related to the detector behaviour, simulation and selection procedure.

The systematic uncertainties of the first class have been evaluated from the Monte Carlo statistics and by varying the parameters describing the $b$ and $c$ fragmentation and the branching fractions of the processes $\mathrm{b} \rightarrow \mathrm{c} \rightarrow \ell, \mathrm{b} \rightarrow \mathrm{X}_{\mathrm{c}} \ell \nu$ and $\mathrm{b} \rightarrow \overline{\mathrm{c}} \rightarrow \ell$. The ranges of variation are shown in Table 3 , which also gives the corresponding uncertainties on the expected number of background Monte Carlo events. The lepton spectrum in the $\mathrm{b}$ rest frame for $\mathrm{b} \rightarrow \mathrm{X}_{\mathrm{c}} \ell \nu$ transitions has been reweighted according to the 


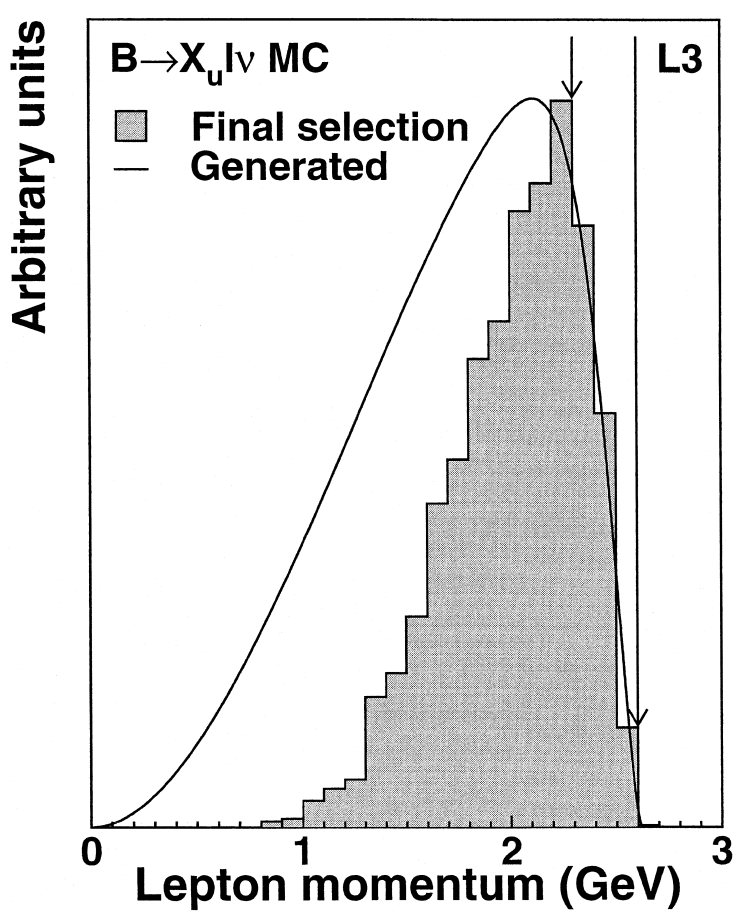

Fig. 3. The Monte Carlo lepton momentum spectrum for the $\mathrm{B} \rightarrow \mathrm{X}_{\mathrm{u}} \ell \nu$ transitions in the $\mathrm{B}$ rest frame generated as described in the text. The curve shows the generated spectrum and the histogram the spectrum after the final selection. selection for the expected $\mathrm{B} \rightarrow \mathrm{X}_{\mathrm{u}} \ell \nu$ transitions. The arrows show the momentum range of the $\Upsilon(4 \mathrm{~S})$ measurements.

ISGW model [31] varying the fraction of $\mathrm{D}^{\star \star}$ production in semileptonic $\mathrm{b}$ decays between $11 \%$ and $32 \%$ [27]. Lepton misidentification has been evaluated by varying by $\pm 5 \%$ the amount of selected hemispheres not belonging to any of the $\mathrm{b} \rightarrow \mathrm{c} \rightarrow \ell$, $\mathrm{b} \rightarrow \mathrm{X}_{\mathrm{c}} \ell \nu, \mathrm{b} \rightarrow \overline{\mathrm{c}} \rightarrow \ell$ and $\mathrm{c} \rightarrow \ell$ classes.

The evaluation of the second class of uncertainties follows, apart from the Monte Carlo statistics, from varying the same $b$ fragmentation parameter considered for the background case, from the elimination of the corrections described above to the JETSET 7.4 Monte Carlo for the pion exclusive decay rate and the pion momentum spectrum, and from the reweighting of the lepton spectrum according to the ISGW model [31]. Another uncertainty has been attributed to the efficiency by varying the $b$ baryon efficiency between 0.5 and 1.5 of the calculated meson one. The fraction of baryons in $b$ hadronisation at LEP has been assumed to be $13.2 \%$ [32]. Table 3 summarises the systematic uncertainties on the total efficiency quoted above.

The Poissonian likelihood used in the determination of the branching fraction has been recalculated 10000 times varying the number of expected background Monte Carlo events and the value of the signal efficiency, within their uncertainties. From the distribution of the resulting branching fractions, a systematic uncertainty of $1.67 \times 10^{-3}$ has been inferred.

The described normalisation of the Monte Carlo to the data depends itself on the content of $\mathrm{B} \rightarrow \mathrm{X}_{\mathrm{u}} \ell \nu$ transitions in the former sample. By varying this content between zero and twice its default value, a variation of $0.10 \times 10^{-3}$ on the measured branching fraction is observed and is added in quadrature to the previous systematic uncertainties. This estimate also covers the lack of charmless semileptonic decays of

Table 3

Contributions to the systematic uncertainties as described in the text. The corresponding uncertainties on the number of the background Monte Carlo events, $\Delta \mathrm{N}$, and on the signal efficiency, $\Delta \varepsilon$, are presented. The percentage variations of the branching fractions are relative

\begin{tabular}{llrll}
\hline Source & Variation & $\Delta \mathrm{N}$ & Source & $\Delta \varepsilon$ \\
\hline MC statistics & & 15 & MC statistics & $0.06 \%$ \\
$\mathrm{~b}$ fragmentation & $0.705<\left\langle x_{\mathrm{E}}^{\mathrm{b}}\right\rangle<0.713$ & 8 & b fragmentation & $0.00 \%$ \\
$\mathrm{c} \mathrm{fragmentation}$ & $0.472<\left\langle x_{\mathrm{E}}^{\mathrm{c}}\right\rangle<0.489$ & 0 & Exclusive $\pi$ rate & $0.18 \%$ \\
$\mathrm{Br}(\mathrm{b} \rightarrow \mathrm{c} \rightarrow \ell)$ & $\pm 5 \%$ & 3 & ISGW model & $0.04 \%$ \\
$\mathrm{Br}\left(\mathrm{b} \rightarrow \mathrm{X}_{\mathrm{c}} \ell \nu\right)$ & $\pm 5 \%$ & 17 & $\pi$ spectrum & $0.25 \%$ \\
$\mathrm{Br}(\mathrm{b} \rightarrow \mathrm{c} \rightarrow \ell)$ & $\pm 20 \%$ & 0 & $\mathrm{~b}$ baryons & $0.40 \%$ \\
$\mathrm{~b} \rightarrow \mathrm{X}_{\mathrm{c}} \ell \nu$ model & $11 \%<\mathrm{D}^{\star \star}<32 \%$ & 31 & & \\
lepton misidentification & see text & 4 & & \\
Total & & 40 & & $0.51 \%$ \\
\hline
\end{tabular}


b baryons in the background Monte Carlo sample used in the normalisation.

The last class of systematic effects, those depending on the simulation of the variables used in the analysis and on the detector performance, have been estimated by observing the changes of the measured branching fraction for the following cases:

- elimination of one cut at a time from the final analysis, fixing it at its stage 1 value;

- reweighting bin by bin one variable at a time in the background Monte Carlo at stage 2 to its value in the data after subtracting from this bin its measured content of $\mathrm{b} \rightarrow \mathrm{X}_{\mathrm{u}} \ell \nu$. Fig. 4 shows the results of these two checks.

- a simultaneous linear loosening and tightening of all the cuts in the range reported in the last column of Table 2. The results of this test are displayed in Fig. 5 and show the stability of the measurement over one order of magnitude of the considered data statistics.

From these studies, a further systematic uncertainty of $0.50 \times 10^{-3}$, including the uncertainties arising from the selection criteria and the detector behaviour and simulation, has thus been attributed to the measured branching fraction.

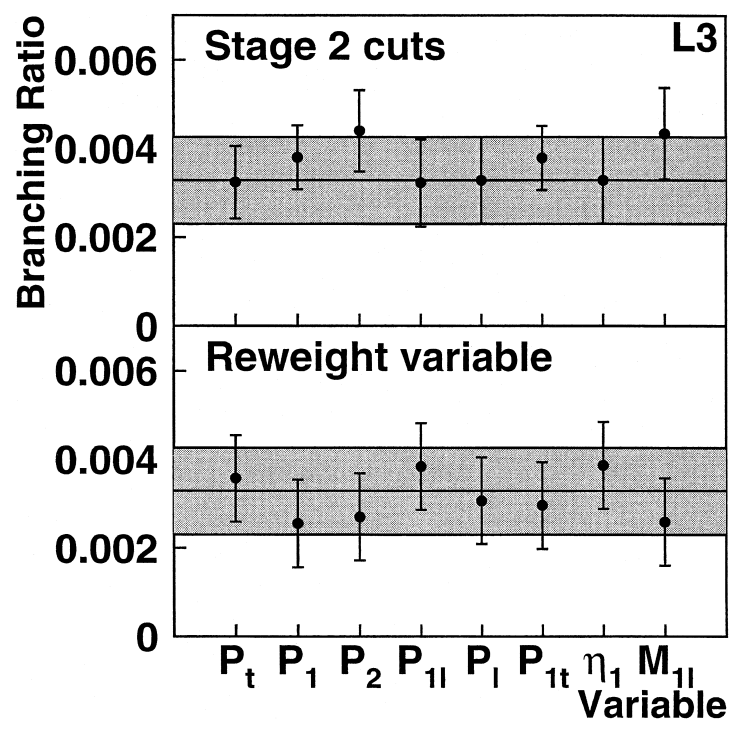

Fig. 4. Variation of the measured $\mathrm{b} \rightarrow \mathrm{X}_{\mathrm{u}} \ell \nu$ branching fraction after the cross checks described in the text for the variables used in the final selection. Only statistical uncertainties are shown. The band indicate the statistical uncertainty on the central value.

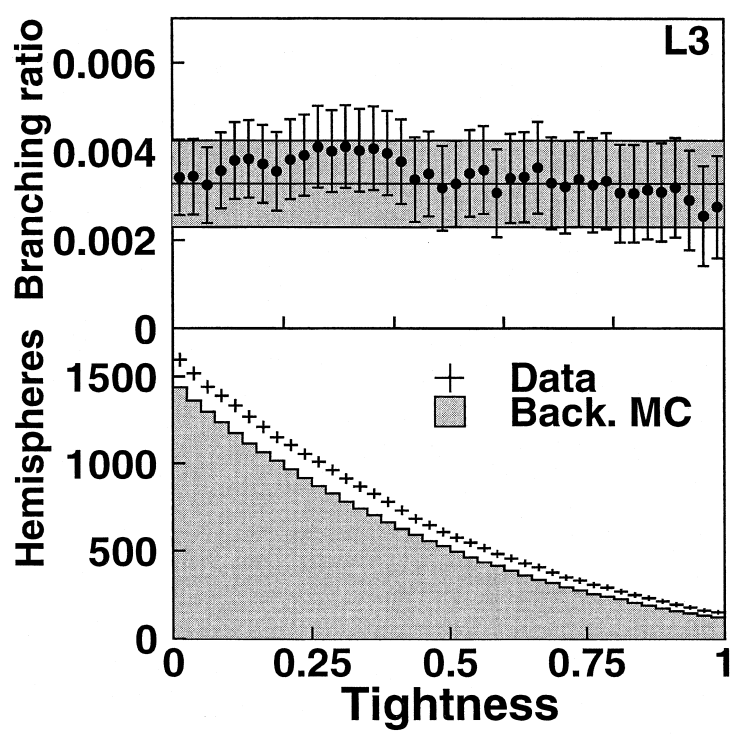

Fig. 5. The measured $\mathrm{b} \rightarrow \mathrm{X}_{\mathrm{u}} \ell \nu$ branching fraction (top figure) calculated from the number of selected data and the background Monte Carlo hemispheres (bottom figure) as a function of a linear tightening of all the cuts. Only statistical uncertainties are shown. The band in the top figure shows the statistical uncertainty on the central value, corresponding to the centre of the variation interval

Adding all systematic uncertainties in quadrature, the result of the measurement of the inclusive charmless semileptonic branching fraction of beauty hadrons, $\mathrm{b} \rightarrow \mathrm{X}_{\mathrm{u}} \ell \nu$, is:

$\operatorname{Br}\left(\mathrm{b} \rightarrow \mathrm{X}_{\mathrm{u}} \ell \nu\right)=(3.3 \pm 1.0 \pm 1.7) \times 10^{-3}$.

The first uncertainty is statistical and the second is systematic.

\section{Cross checks of the result}

A first cross check of the result presented above is given by the three tests performed to estimate the detector and simulation uncertainties and from that on the normalisation, as the value of the measured branching ratio remains sufficiently stable within its statistical uncertainty (Figs. 4 and 5.)

Nonetheless, it is desirable to obtain a determination of the branching fraction under investigation independently of the background Monte Carlo normalisation. This is difficult from the investigation of the distributions of the eight kinematic variables 


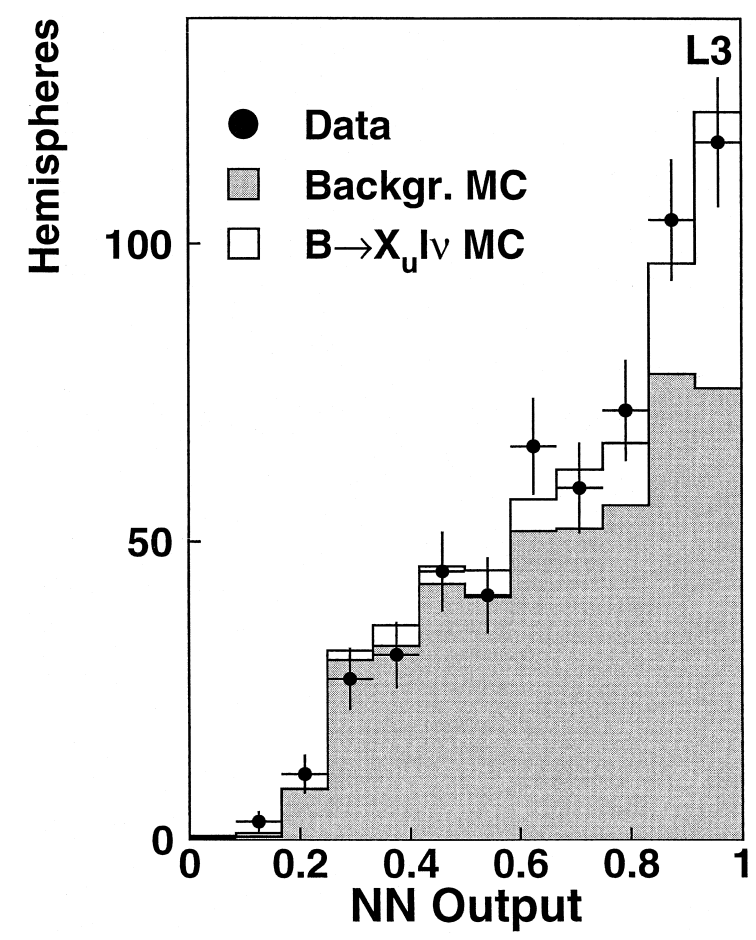

Fig. 6. The neural network output distribution for the hemispheres selected by the final selection criteria for the data, the background and the signal Monte Carlo samples. The normalisation of the Monte Carlo samples comes from the fit to the data.

after the final cuts as they show a similar behaviour for both the expected background and signal. Instead, these variables have been used as input to an artificial neural network with two hidden layers of 14 and 8 nodes each, making use of the JETNET 3.0 program [33]. This neural network was trained on subsets of the signal and background Monte Carlo samples at stage 2 , and its output has been analysed for the complementary subsets. The output distribution of the neural network for the hemispheres selected by the final selection in these signal and background Monte Carlo subsamples was then fit to the data using a binned Poissonian likelihood method. Two parameters have been left free in the fit, namely the branching ratio under investigation and the number of background events. Using this procedure one obtains the result:

$\mathrm{Br}\left(\mathrm{B} \rightarrow \mathrm{X}_{\mathrm{u}} \ell \nu\right)=(4.2 \pm 1.2) \times 10^{-3}$.
This result is compatible with the measurement obtained above, and has a comparable systematic uncertainty.

The output distribution of the neural network is shown in Fig. 6, together with the result of the fit. It shows a different behaviour for the background and signal Monte Carlo events, along with a clear excess of data in the expected signal region.

\section{Conclusions}

Inclusive charmless semileptonic transitions of $b$ hadrons have been observed at LEP and their branching fraction has been measured to be:

$\operatorname{Br}\left(\mathrm{b} \rightarrow \mathrm{X}_{\mathrm{u}} \ell \nu\right)=(3.3 \pm 1.0 \pm 1.7) \times 10^{-3}$.

The first uncertainty is statistical and the second is systematic.

With the formula given in Refs. $[13,14]$ and the value of the $\mathrm{b}$ hadron lifetime $\tau_{\mathrm{b}}=1.549 \pm 0.017 \mathrm{ps}$ [29], this measured $b \rightarrow X_{u} \ell \nu$ branching fraction yields a value for the modulus of the CKM matrix element $\mathrm{V}_{\mathrm{ub}}$ of:

$\left|\mathrm{V}_{\mathrm{ub}}\right|=\left(6.0_{-1.0-1.9}^{+0.8+1.4} \pm 0.2\right) \times 10^{-3}$,

where the first uncertainty is statistical, the second systematic and the third follows from the theory uncertainty quoted in Ref. [14]. This measurement of $\left|V_{\mathrm{ub}}\right|$ made at the $\mathrm{Z}$ pole is less affected by the theoretical modelling of the $b \rightarrow u$ transitions than previous ones at the $\Upsilon(4 \mathrm{~S})$ resonance. It is compatible both with them and with a similar measurement at LEP [15].

\section{Acknowledgements}

We wish to express our gratitude to the CERN accelerator divisions for the excellent performance of the LEP machine. We acknowledge the contributions of all the engineers and technicians who have participated in the construction and maintenance of this experiment.

\section{References}

[1] N. Cabibbo, Phys. Rev. Lett. 10 (1963) 531.

[2] M. Kobayashi, K. Maskawa, Prog. Theo. Phys. 49 (1973) 652. 
[3] S.L. Glashow, Nucl. Phys. 22 (1961) 579.

[4] A. Salam, in: N. Svartholm (Ed.), Elementary Particle Theory, Almqvist and Wiksell, Stockholm, 1968, p. 367.

[5] S. Weinberg, Phys. Rev. Lett. 19 (1967) 1264.

[6] L. Wolfenstein, Phys. Rev. Lett. 51 (1983) 1945.

[7] CLEO Collaboration, R. Fulton et al., Phys. Rev. Lett. 64 (1990) 16.

[8] CLEO Collaboration, J. Bartelt et al., Phys. Rev. Lett. 71 (1993) 4111.

[9] ARGUS Collaboration, H. Albrecht et al., Phys. Lett. B 234 (1990) 409

[10] ARGUS Collaboration, H. Albrecht et al., Phys. Lett. B 255 (1991) 297.

[11] CLEO Collaboration, A. Bean et al., Phys. Rev. Lett. 70 (1993) 2681.

[12] CLEO Collaboration, J.P. Alexander et al., Phys. Rev. Lett. 77 (1996) 5000.

[13] N.G. Uraltsev, Int. Jour. of Mod. Phys. A 11 (1996) 515.

[14] I. Bigi et al., Ann. Rev. Nucl. Part. Sci. 47 (1997) 591.

[15] ALEPH Collaboration, R. Barate et al., Preprint CERNEP/98-067, 1998.

[16] L3 Collaboration, B. Adeva et al., Nucl. Instr. Meth. A 289 (1990) 35.
[17] L3 Collaboration, O. Adriani et al., Physics Reports 236 (1993) 1.

[18] M. Acciarri et al., Nucl. Instr. Meth. A 351 (1994) 300.

[19] A. Adam et al., Nucl. Instr. Meth. A 383 (1996) 342.

[20] T. Sjöstrand, Computer Physics Commun. 82 (1994) 74.

[21] T. Sjöstrand, Preprint CERN-TH.7112/93, 1993.

[22] G. Altarelli et al., Nucl. Phys. B 208 (1982) 365.

[23] G. Burdman, J. Kambor, Phys. Rev. D 55 (1997) 2817.

[24] R. Brun et al., GEANT 3, CERN DD/EE/84-1 (Revised), September 1987.

[25] H. Fesefeldt, Preprint RWTH Aachen PITHA 85/02, 1985.

[26] C. Peterson et al., Phys. Rev. D 27 (1983) 105.

[27] The LEP Collaboration, Nucl. Instr. Meth. A 378 (1996) 101.

[28] The LEP Collaboration, The LEP Electroweak Working Group and The SLD Heavy Flavour Group, D. Abbaneo et al., Preprint CERN-PPE/97-154, 1997.

[29] L3 Collaboration, M. Acciarri et al., Phys. Lett. B 416 (1998) 220.

[30] L3 Collaboration, B. Adeva et al., Z. Phys. C 51 (1991) 179.

[31] N. Isgur et al., Phys. Rev. D 39 (1989) 799.

[32] Particle Data Group, Phys. Rev. D 54 (1996) 1.

[33] C. Peterson et al., Comp. Phys. Comm. 81 (1994) 185. 